\title{
Sensitive ligand-based protein quantification using immuno-PCR: A critical review of single-probe and proximity ligation assays
}

\author{
Marcus Celik Hansen ${ }^{1}$, Line Nederby ${ }^{1}$, Mads Okkels-Birk Henriksen², Maria Hansen ${ }^{1}$, and \\ Charlotte Guldborg Nyvold ${ }^{1}$ \\ ${ }^{1}$ HemoDiagnostic Laboratory, Department of Hematology, Aarhus University Hospital, Aarhus, Denmark and \\ ${ }^{2}$ Department of Haematology, Copenhagen University Hospital, Herlev, Denmark.
}

BioTechniques 56:217-228 (May 2014) doi 10.2144/000114164

Keywords: immuno-quantitative PCR, proximity ligation assay, immuno-PCR, multiplex protein analysis, aptamers

Quantitative PCR (qPCR) of reverse-transcribed mRNA has revolutionized gene expression analyses. qPCR analysis is based on the prevalent assumption that mRNA transcript numbers provide an adequate measure of specific biomarker expression. However, taking the complexity of protein turnover into account, there is a need to correlate qPCR-derived transcriptional patterns with protein translational patterns so as to not leave behind important pathobiological details. One emerging approach in protein analysis is PCR-coupled protein quantification, often denoted as immuno-PCR (iPCR), which targets soluble proteins. Here we review recent trends and applications in iPCR assays that may bridge the gap between classical enzyme-linked immunosorbent assays and mass spectrometry methodologies in terms of sensitivity and multiplexing.

The practical implications of gene expression analysis in biomedical research are clear-cut. Real-Time quantitative PCR ( $\mathrm{PPCR}$ ) has offered immense assistance in diagnosis and prognosis based on comparative mRNA levels. However, it has been shown that the transcriptional level does not necessarily correlate with protein expression (1-3), with one recent study even reporting an inverse relationship of $\mathrm{mRNA}$ and protein expression in breast cancer tumor prognosis (4). A noteworthy large-scale gene expression study has disproven the idea of a global correlation in mRNA and protein turnover and confirmed the overall variability in transcriptional and translational patterns (5). There is no such thing as a static proteome or transcriptome, emphasizing that we observe snapshots of the cellular state in time and place. Bearing this in mind, protein expression can give clues about pathogenesis that are not apparent at the transcriptional level or provide new insights through correlation analyses. In some cases, for example detection of plasma biomarkers derived from peripheral blood samples, protein quantification techniques are crucial.
In the field of large-scale protein analysis there is no way around mass spectrometry (MS). Powerful MS techniques have contributed to proteomics in a variety of ways, offering quantitative approaches. Relative or absolute quantification analyses can utilize either differential stable isotope labeling or label-free methods. (For more information on comparing gel-based methods to MS, see the comprehensive introductory review in Reference 6.)

Interesting as it is, while MS plays an important role in proteome exploration, the technology is still quite inaccessible for many researchers, owing either to economical considerations or the need for specialized skills to perform the analyses. In addition, the method can be inappropriate for a given experimental setup. So, what alternatives, other than ELISAs and blots, are there for the rest of us?

Here we review non-spectrometry ligand-based protein quantification using PCR-coupled approaches. Each of the methods discussed presents a different set of obstacles; thus, an introductory review that also takes an objective and critical view of PCR-coupled protein analysis methods is needed. In the following discussion, we point out some of the shortcomings and practical uses of various immuno-quantitative PCR assays. Although each has different strengths and aims, high sensitivity and specificity is essential in any successful quantification assay.

\section{Merging immunoassays and quantitative PCR}

The founding ideas behind coupling PCR and immunoassays go back more than 20 years (7). In a beautiful layout, Sano et al. used a previously developed streptavidin-protein A chimera (8) as a linker between a DNA segment and a monoclonal antibody specific for immobilized bovine serum albumin. Termed immuno-PCR (iPCR), the basic concept has changed little since then, and the other PCR-coupled methods described here are essentially built on the same principles. Interestingly, this sensitive antigen detection system was described only a year after Holland et al. suggested the use of labeled hydrolysis probes in PCR (9)-the very same technology that revolutionized gene 


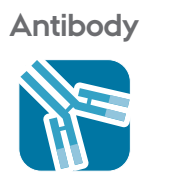

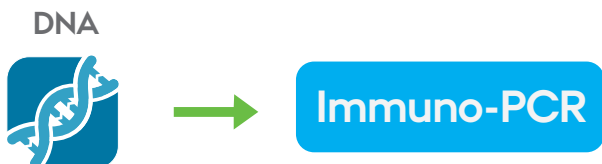

\section{Immuno Assay \\ Polymerase Chain Reaction \\ A}

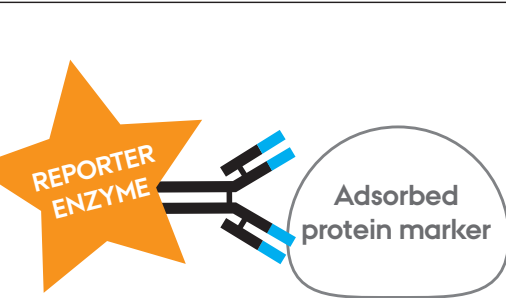

B Traditional Enzyme-linked immunosorbent assay

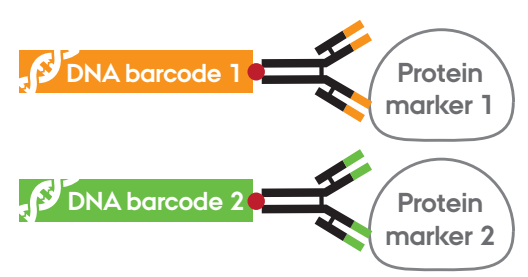

Multiplex Immuno-PCR

Figure 1. The difference between iPCR and enzyme-linked immunoassays. (A) iPCR merges the two domains of antibody and oligonucleotides, where the antibodies supply the affinity and specificity, and the nucleic acid functions as the reporter, in contrast to ELISA (B). (C) Unique barcode identifiers can provide quantitative information from different biomarkers in a single multiplex assay.

expression analysis but also to some extent pushed translational quantification out of focus.

The enhanced limit of detection of iPCR compared to ELISA is reported to be in the range of a modest 100-fold to a staggering $10^{5}$-fold (10-12). While the enhanced sensitivity is unambiguously ascribed to quantitative PCR, simply stating that the detection limit of iPCR is increased manifold in contrast to its enzyme-linked counterpart is an oversimplification that cannot be deduced a priori, as will be explained in detail later. IPCR is methodologically related to ELISA, where special measures have to be taken to harness the advantages of increased readout sensitivity.

Antibodies or other types of ligands used in PCR-coupled immunoassays are directly or indirectly barcoded with a synthetic nucleic acid strand (Figure $1 \mathrm{~A}$ ) instead of being conjugated to a reporter enzyme (Figure $1 \mathrm{~B}$ ) such as horse radish peroxidase or alkaline phosphatase. The barcode analogy is useful when considering one of the obvious advantages: unique identifiers can provide quantitative information from different biomarkers in a single run (Figure 1C).

Not all PCR-coupled immunoassays require immobilization of the target proteins. A proximity ligation assay (PLA), described in the following section, can detect specific proteins or protein-protein interactions directly in a homogenous solution. The nature of the signal-to-noise ratio (SNR) is also different compared to other iPCR assays, and this issue will be treated separately.

\section{Proximity ligation assay (PLA)}

Ultra-sensitive detection of plateletderived growth factor by PLA was first described in 2002 by a Swedish research group (13). Swedish researchers still constitute the frontline in refining this technology. The PLA ingeniously utilizes two DNA aptamer-based proximity probes, each containing a sequence extension to which a common complementary connector oligonucleotide binds, pairing the probes in close proximity (Figure 2). After probe pair ligation, the DNA is amplified by qPCR for quantitative evaluation-with the protein content now superfluous. Alternatively, and now common practice, two antibodies (mono- or polyclonal), can substitute for the binding aptamers in the assay. An antibody-dependent PLA approach is exploited in commercially available pre-conjugated kits (Life Technologies, Carlsbad, CA; Olink Bioscience, Uppsala, Sweden). Simplifying the setup, a single biotinylated polyclonal antibody population raised against a full-length protein immunogen can be split into two tubes for conjugation with ether a
5'- or 3'-oligo. Although selecting two monoclonal antibodies in order to get more specific PLA probes may be more cumbersome initially, this strategy can provide increased reliability and reproducibility in a standardized clinical environment.

An advantage of protein detection and quantification by ligand-based PCR methods is the potentially low consumption of antibodies. Gullberg et al. postulate that more than 100,000 PLAs can be performed with just one microgram of antibody (12). Furthermore, the very low limit of detection enables analyses on sparse samples. It has been shown feasible to use as little as one microliter (14) of patient plasma material. It must be noted that very low consumption of biological sample or antibody is not always desirable, as this increases the standard deviation-obviously a limiting factor of reproducibility. Although often presented as an advantage of PLA, the minute consumption of antibody and biological sample represents an Achilles' heel of the assay: too high a probe concentration will lead to a decrease in the SNR due to nonspecific background ligations, and too high a protein concentration will cause probe depletion.

\section{Target adsorption with}

\section{solid phase PLA}

Immobilization of the target protein, as performed in solid phase PLA (SP-PLA), can be necessary to obtain sufficient sensitivity and a wider dynamic range where homogenous assays fail, as reported by Zhu et al. (15). In microparticle-based SP-PLA, a polyclonal antibody can be aliquoted into three pools - two for affinity probe incorporation and one for immobilizing target proteins to paramagnetic particles (16). Another possibility is using streptavidincoated tubes or wells as solid supports. Darmanis et al. demonstrated high correlation between PCR threshold cycle $(\mathrm{Cq}$ value) and interleukin-8 concentration in a two-fold plasma dilution with a coefficient of determination $\left(R^{2}\right)$ of 0.997 in the microparticle setup (16). This study also showed comparable sensitivities between the microparticle-based protocol and one involving coated polycarbonate tubes for target protein anchoring.

Introducing washing steps can minimize possible interference and 
inhibition by other components of the cell lysate and reduce background signal from unbound probes. But if washing steps are included in the proximity ligation assay, why use a two-probe system instead of one probe? Increased specificity gained by multiple target recognitions is an important feature, but several sensitive methods rely on a single probe to which additional specificity is provided through capture antibodies. These methods are collectively referred to as iPCR. The use of two probes in PLA complicates the setup of novel analyses. Not only do they have to target different epitopes of the same protein, as in sandwich ELISA, but they must also be proximaly situated in order to ligate successfully. In return, increased specificity is possible.

\section{Extended variants of mono-probe iPCR assays}

Immunodetection amplified by T7 RNA polymerase (IDAT) assay is the basis for another branch of the PCR-coupled immunoassay family. Here, a doublestranded DNA oligonucleotide containing the T7 promoter is conjugated to the target-specific ligand (17). Amplification using phage T7 RNA polymerase renders thermocycling in iPCR obsolete and provides linear product generation. This scheme requires other means of signaling than those using Taq polymerase 5'-3' exonuclease activity, where the signal is obtained by a reporter dye, freed from its quencher situated in close proximity on the hydrolysis probe. Here, RNA-intercalating dyes can be incorporated as reporter molecules and read on a fluorometer $(18,19)$. This method, superseding the earlier gel-based isotope detection by Zhang et al. (17), is known as fluorescent amplification catalyzed by T7 polymerase technique, or FACTT for short. The downside of IDAT/FACTT assays is the limitation in direct scalability and multiplexing utilizing dyes with specific absorption maximums. One possible expansion we suggest could involve hybridization on a chip as exploited with DNA microarrays. Linear signal generation in IPCR and PLA can also be accomplished by isothermal rolling circle amplification (20), where a circular amplicon serves as template for continuous product generation by the polymerase.

Reported molar detection ranges for the previously discussed methods are shown in Figure 3.

\section{Methodological considerations}

\section{Setting up iPCR in the laboratory}

iPCR will not gain widespread use unless it is easy to implement in a clinical laboratory, taking workload and cost-benefit into consideration. This section will describe some basic and practical details for setting up the analysis. If ELISA and qPCR are already performed routinely in the laboratory, setting up the assay is essentially confined to the construction of iPCR probes from antibodies with adequate antigen specificity and oligonucleotides with sufficient PCR amplification efficiency, while keeping the existing protocols relatively intact.

\section{Probe construction}

Construction of iPCR probes requires (i) an antibody with high affinity and speci- ficity for the target protein, (ii) an amplifiable synthetic DNA sequence, and (iii) the successful combination of the two.

The synthetic DNA sequence consists of flanking primer annealing regions and an optional sequence complementary to a hydrolysis probe in between.

As the template sequence can be arbitrarily selected, the design can be based on purely random sequences, on a shuffle of existing forward and reverse primers already used in the laboratory, or on already published sequences (e.g., the set targeting foot-and-mouth disease virus reported by Ding et al. in Reference 21). However, it is recommended to avoid primer sequences likely to amplify genomic DNA, as this will affect the results.

The strong non-covalent interactions between streptavidin and biotin can be exploited as a simple means of probe construction. If a biotinylated antibody is available, the stepwise assembly procedure is straightforward. In a biotin-streptavidin-biotin self-assembly strategy described by Darmanis et al. for formation of proximity probes (22), the modified oligos and strepavidin homotetramer are incubated in a 1:1 molar ratio initially, followed by the addition of biotinylated antibodies in a 1:2 ratio. One might expect the optimal ratio of oligos and antibody bound through a tetravalent streptavidin complex to be 4:1. However, it has been shown that bivalent linkage of biotinylated antibody and oligo is most likely to occur (23).

Obtaining a biotinylated variant of an antibody directly from the supplier may not be possible, whereas a wide range of oligo modifications are readily commer-
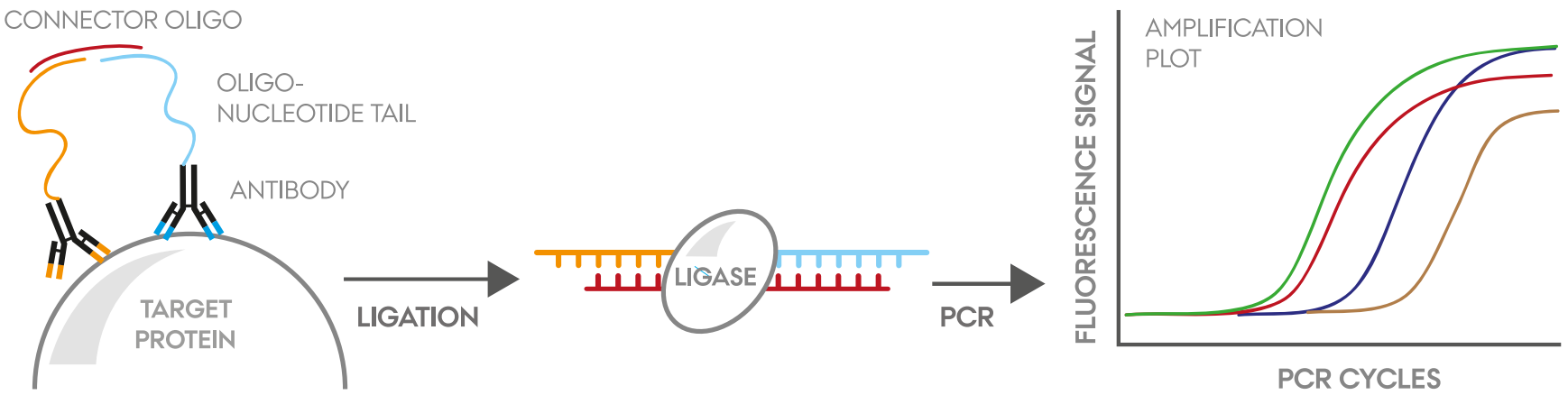

Figure 2. The proximity ligation assay-one type of PCR coupled protein quantification analysis. The proximity ligation assay and other similar ligand-based methods couple Real-Time PCR with target protein detection and quantification. In PLA, two antibodies with oligonucleotide tails raise the local concentration through ligand epitope recognition on the protein of interest. This enables amplification following connector-oligo linkage and enzymatic ligation of DNA strands. 


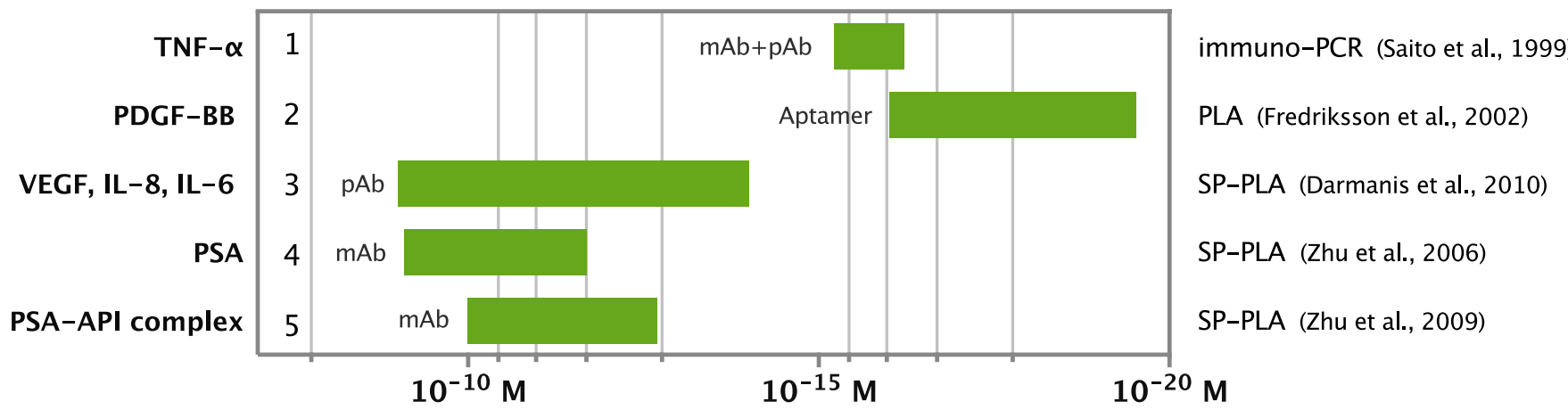

Figure 3. Reported detection ranges for ligand-based PCR methods. Molar detection range was calculated from mass concentration based on the PSA-API complex molecular weight (MW) of approximately $82 \mathrm{kDa}$, PSA MW of approximately $30 \mathrm{kDa}$, and TNF- $\alpha$ MW of approximately $17 \mathrm{kDa}$. Other studies listed molar concentrations.

cially available, such as a 5' or 3' biotin moiety attached through a carbonchain spacer. Several commercial antibody biotinylation kits are available. The efficiency of incorporation can be estimated spectrophotometrically with HABA dye (4'-hydroxyazobenzene2-carboxylic acid) (24). Alternatively, a biotinylated secondary antibody eliminates the need for modifying specific antibodies and is practical for preliminary antibody testing. This scheme was described by Zhou et al. (25) in 1993, but it hinders direct multiplexing by eliminating the possibility of unique DNA sequence conjugation to targetspecific antibodies.

Once an oligo that can be amplified in PCR with adequate efficiency is successfully conjugated to the antibody, the optimal dynamic range is determined. As in ELISA, one can perform checkerboard titrations to find the optimal antibody concentration, where a direct iPCR constitutes the simplest layout for optimization (Figure 4).

Collecting residual oligos prior to PCR If PCR is carried out in a separate tube, the oligos have to be freed from adsorption to be transferred to the PCR tubes. Simply heating the samples is sufficient to release DNA (26). Alternatively, proteinase (e.g., Proteinase K from Tritirachium album, Sigma-Aldrich, St. Louis, MO) can be added to each sample well to degrade immobilized target protein, antibodies, and other proteinaceous contents. Complete heat inactivation of proteinases before adding polymerase is, of course, necessary.

\section{Notes on specificity}

Strikingly, a considerable number of the published articles involving iPCR do not include negative biological controls in the assay. In clinical use, an assay is not valid if the SNR does not represent a true difference between a pathological observation and a control sample that is derived from a validated negative cell line or healthy donors. This requirement, we argue, represents one of the major bottlenecks of clinical iPCR implementation, as a consequence of using antibodies in assays without spatial resolution. If reported sensitivities merely represent the widest possible range of detection without questioning the validity of the target specificity, which is encountered in proof-of-concept articles, the published SNR is of no use to clinical laboratories interested in implementing the assays.

One can also argue that simply measuring the dynamic range by a more sensitive method such as qPCR is a poor improvement, given that the SNR ultimately reveals to be equivalent to colorimetric immunoassays. That is, the level of background noise the immunoassay is clearly a limiting factor, even if qPCR is used. Hence, steps that effectively minimize noise contributions are important to take advantage of the increased sensitivity. As the causes of these contributions differ according to assay type, reported examples of SNR improvements are considered in the following sections. We first turn to PLA, as the nature of the SNR for that method differs from other iPCR assays.
Signal-to-noise ratio in

homogenous PLA

Signal contribution in homogenous PLA stems from both proximal ligated probe pair DNA and randomly ligated strands in bulk solution (27) (Figure 5). This inevitably leads to a lower intrinsic SNR and is one of the drawbacks of the original homogenous variant. Lowering the probe concentration reduces the likelihood of random ligation (28) but narrows the dynamic interval of detection (29). Also, the extent of background contributions must, theoretically, be a function of incubation time and thermal energy in the solution. Due to the nature of the signal, a linear standard curve cannot be expected, but-as is true for other immunoassays - a local linear range is approximated.

Various methods have been tried to reduce background noise in PLA. Fredriksson et al. described the use of thermostable ampligase, which displays low activity below $30^{\circ} \mathrm{C}$, for more consistent ligation conditions (Supplementary Material for Reference 27). In asymmetric PLA, connector oligonucleotide binding affinity towards the probe strands is weakened in one end, reducing the probability of targetindependent ligation (29). Triple-specific proximity ligation assay (3PLA) lowers background signal by using weakly hybridizing oligonucleotides to temporarily block unbound probes and increases specificity through triple recognition of three separate target probes (30). Landegren et al. reported a 100-fold increase in sensitivity compared to standard homogenous PLA when assaying recombinant VEGF (22). The latter study from the group 


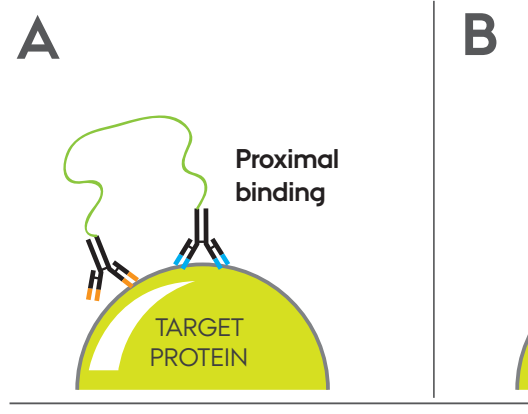

TRUE POSITIVE

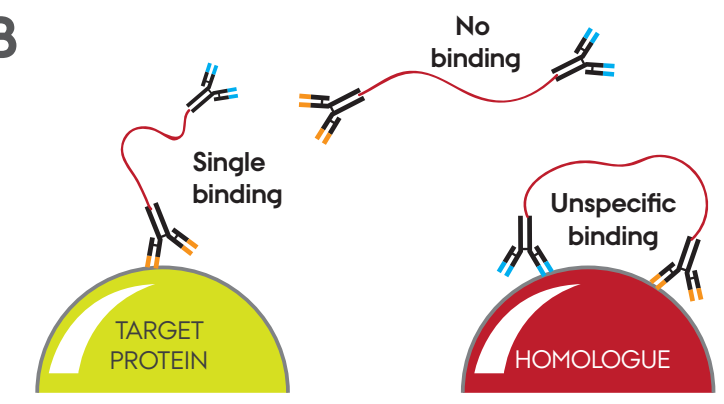

FALSE POSITIVES

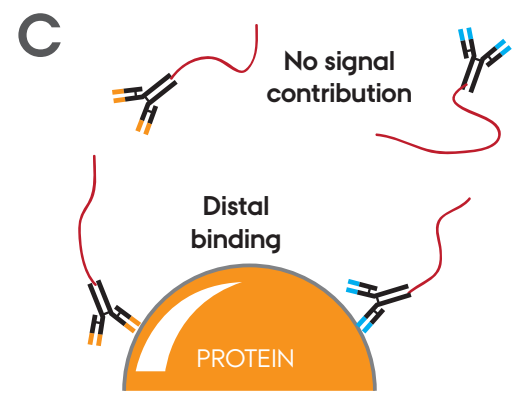

TRUE/FALSE NEGATIVES

Figure 5. Signal contributions in simple homogenous PLA. True positives are a desired contribution to signal intensity and reflect specificity (A), whereas false positives from background ligations (B) are unwanted signal contributions and represent an inherent problem of the proximity assay. Specific single probe binding of target protein, ligated to its complement in the bulk solution, can also be classified as a false positive. The problem with nonspecific binding of probes to homologous counterparts is shared with other immunoassays, but is minimized through the need for proximal binding. False positives arising from random ligation are a function of probe concentration, ligation incubation time, and thermal energy. This demands batch-to-batch stringency. True and false negatives (C) arise due to distal binding and unligated probes in bulk solution.

mining 21 target protein levels in 6- and 7-plex assays (36). General concordance between biomarker levels reported in the literature and results from that study were observed.

To our knowledge very few articles concerning multiplex PLA but not involving the Uppsala research group in Sweden appear in the PubMed database (e.g., References 37 and 38). Time will tell if methods based on this principle will have a large impact in the field of proteomicsor even be competitive with the highthroughput of MS.

\section{Emerging iPCR \\ developments}

Flanigon et al., including Landegren, notably involved in the majority of PLA studies, and Cantor, one of the original inventors behind iPCR, present a possible direction for large-scale multiplex protein quantification (39). Here, the two methods converge in a direct comparison of multiple protein targets, displaying similar dynamic ranges over several orders of magnitude. Absolute quantification was performed with competitive PCR, involving co-amplification of a near identical target sequence of known concentration, and PCR amplification products were subsequently analyzed on a MALDI-TOF mass spectrometer.

Open sandwich (OS) ELISA may not be the most widely distributed sandwich flavor, but it was recently added to the list of PCR-coupled immunoassays. In OS-ELISA, immunoglobulin variable region heavy $\left(\mathrm{V}_{\mathrm{H}}\right)$ and light $\left(\mathrm{V}_{\mathrm{L}}\right)$ chain are initially separated with very low interchain binding affinity and are prone to dissociation (40). One is used as a well-anchor, and the other is conjugated to a reporter. Association of the chains is stabilized through antigen binding, and constitutes an indirect sandwich function. In this sense, sandwich is perhaps a slight misnomer. Hasan et al. brought iPCR into OS-ELISA by engineering a streptavidin and single-chain variable fragment (scFv) fusion protein to target osteocalcin (41). Tethering of the stabilized complex to the wells of an ELISA plate was mediated by maltose binding protein fused with $V_{H}$, and PCR-compatibility was made possible by the biotinylated DNA binding properties of streptavidin. OS-ELISA offers an advantage, when small biomarkers are to be analyzed (i.e., biomarkers, where steric hindrance restricts the binding to one paratope alone). The relatively few published articles on OS-ELISA may be reflected in this specialized, but not limiting, scope and the vast supply of antibodies on the market. However, it provides direct means of utilising recombinant antigen-binding fragments generated by phage display in the IPCR assays, as is described by Dong et al. (42).

Other innovations involve digital PLA (43), presumably enabling single molecule detection by rolling circle amplification.

\section{Aptamers - an antidote to}

the antibody problem?

Prior to a protein study, one has to succeed in generating, or obtaining, high affinity antibodies targeting the polypeptide of interest. Finding suitable ligand pairs for a PLA is a critical step, and it can be a daunting task in a multiplex setup. Zhu \& al. tested eight different monoclonal antibodies in combination with a prostate-specific antigen (PSA) binding peptide (15). Half of the antibodies were described as giving a weak or strong response, and the other half gave no response. Alternatively, instead of using hybridomaderived monoclonal or polyclonal antibodies, one can turn to recombinant antibodies generated by phage display techniques.

One promising, but in some respects underdeveloped, alternative to the use of antibodies is the generation of aptamers through systematic evolution of ligands by exponential enrichment (SELEX). RNA-based aptamers were first described more than two decades ago $(44,45)$ but did not make much of an impact (46) despite the simple concept. Today, the term comprises both engineered high affinity molecules derived from single stranded oligonucleotides, DNA, or RNA, as well as polypeptides. Target affinity range is described as low nanomolar to picomolar (47-49) but is often stated without further reference to supporting literature.

Immense interest in the use of aptamers is now clearly evident in the scientific community (48) for a diverse array of research methods such as flow cytometry (50), cancer-related tyrosine kinase inhibition (51), in vivo cancer imaging models (52), affinity chroma- 
tography, and cytotoxic drug delivery (53). The aptamer offers the advantage of small size, lately showing its worth in super-resolution microscopy (54), fitting into nooks and crannies of the target molecule. The sheer number of scientific articles based on aptamer research indicates broad deployment and technological maturation at a fast pace and a growing general interest. So what and where are the drawbacks?

The common denominator of the various SELEX flavors is the random oligonucleotide library as a starting pool. This can either be generated de novo or be obtained from a supplier, considering the relative low cost of oligonucleotide synthesis. From the point where the library is incubated the with target of interest, the procedure of selection diverges into a plethora of different reported SELEX approaches. However, reviews have so far have mainly focussed on the potential clinical and therapeutical applications, resulting in a somewhat overall narrow scope. We refer to Djordjevic (55), one of the few reviews that try to correct this to some degree biased weighting. Empirical evaluations in the laboratory and methodological comparisons between SELEX methods are needed in order to

\section{Thermo}

S C I ENT I F I C

\section{Trust NanoDrop} measurements

No matter the sample concentration or volume, scientists worldwide rely on the patented Thermo Scientific ${ }^{\mathrm{TM}}$ NanoDrop ${ }^{\mathrm{TM}}$ technology to evaluate their DNA, RNA, and protein samples. Expect reproducible quantity and purity data from microvolume samples without having to perform dilutions or guess which accessory you need. Data you can trust in seconds, backed by over 10,000 peer reviewed citations.

\section{Pipette. Measure. Wipe clean.}

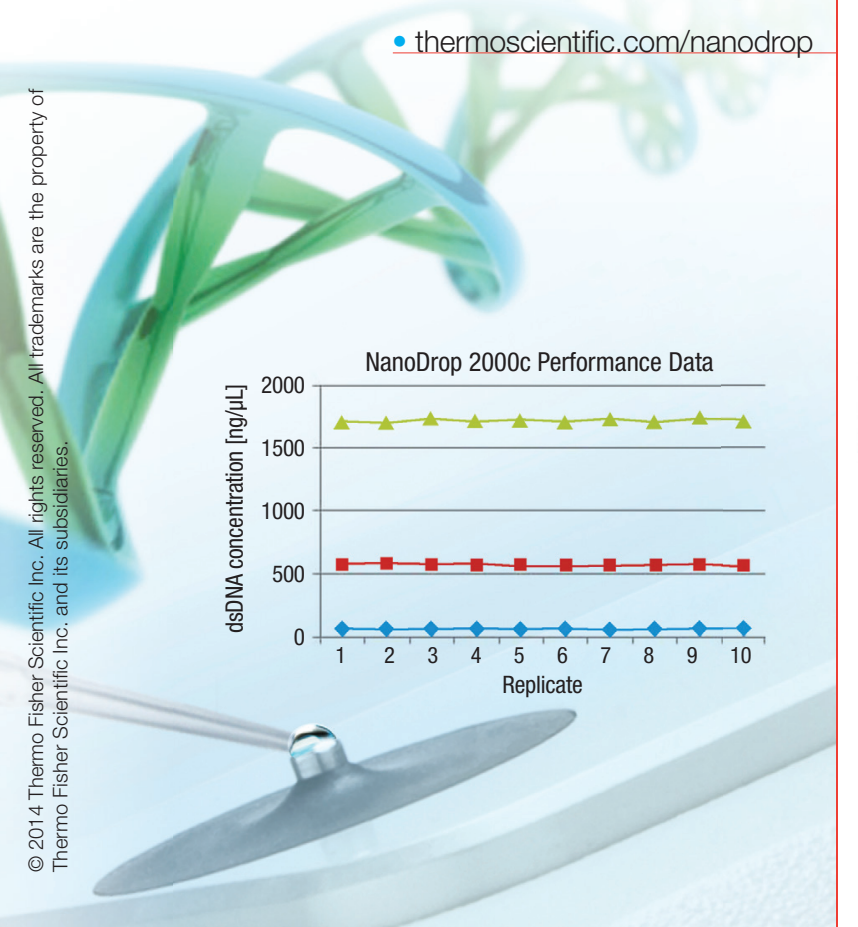

NanoDrop 2000c Spectrophotometer

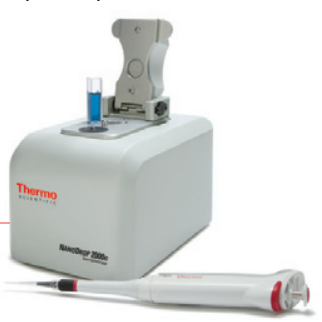

NanoDrop 8000 Spectrophotometer

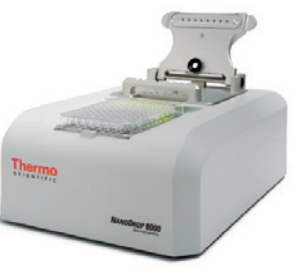

NanoDrop Lite Spectrophotometer

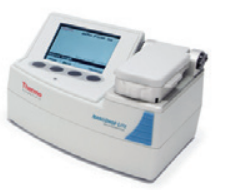

streamline protocols for cost-effective development of novel aptamers for the clinical researcher. Once robust aptamers have been developed thorough sensitivity and specificity testing, the nature of the oligos holds potential for reasonable standardization and sharing between clinical research laboratoriesmeaning reproducible probes for flow cytometry, PLA, and affinity chromatography or ELISA analogues using aptamers as surrogate antibodies. Given the crucial contributions from secondary structure, the affinity of the aptamer ligands must be reevaluated in a given nano-environment. Buffer or temperature change, chaotropic agents, or the addition of linkers and conjugates to the aptamer can, theoretically, easily alter inherent secondary structure. General studies regarding consistency in aptamer efficacy have yet to be published.

\section{Concluding remarks}

In summary, all the immuno-quantitative PCR methods described here offer protein quantification with potential high sensitivity in a variety of ways. Homogenous PLA enables highly specific detection and quantitative analyses directly in cell lysates but requires carefully matched probe pairs. We hope to see more clinical studies in the future for practical and independent evaluation of PLA. The focus among published articles has predominantly centered on proof-of-concept, with little emphasis on clinical research. One particular offspring of the proximity ligation technology has found broad utilization as a research tool in a multitude of studies: in situ PLA, a method for probing protein-protein interactions, represents an unambiguous practical implemented innovation.

Immobilizing target proteins by capture antibodies offers higher specificity, enables washing, and reduces the complexity of the probes. Probes consisting entirely of oligonucleotides, through the virtue of aptamers, can potentially contribute to standardization in clinical settings and ease reproducibility. However, empirical maturation of the concept is much needed to elucidate the practical implications of the aptamers.

If the obvious problems with the specificity and SNR are solved, immunassays in combination with $\mathrm{gPCR}$ have the potential 
to become standard methods for protein quantification. Having learned the lesson from the early days of qPCR, efforts should be made to create instructions and standards for assay reproducibility equivalent to MIQE guidelines (56) at an early stage (e.g., preparation of input material for the qPCR measurements). Standardization of this step and the immunodetection procedures is essential for robust, reliable, and reproducible results as well as for the ability of researchers to critically evaluate and compare published data, thereby avoiding erroneous conclusions. Highly sensitive analysis readout is offered through iPCR, but poses many of the traditional problems common to immunoassays. It cannot be expected a priori that the SNR is improved base on $P C R$ readouts alone.

Implementation at clinical affiliated laboratories is still sparsely reported, despite the fact that more than two decades has passed since this innovative combination of antigen recognition and PCR readout was first reported.

\section{Author contributions}

All authors contributed equally.

\section{Acknowledgments}

We wish to thank senior laboratory assistant Annette Gudmann Hansen (Department of Biomedicine, Aarhus University) for discussions on antibody conjugations and Peter Hokland (Department of Hematology, Aarhus University Hospital) for scientific guidance.

\section{Competing interests}

The authors declare no competing interests.

\section{References}

1. Asplund, A., M. Gry Björklund, C. Sundquist, S. Strömberg, K. Edlund, A. Ostman, P. Nilsson, F. Pontén, and J. Lundeberg. 2008. Expression profiling of microdissected cell populations selected from basal cells in normal epidermis and basal cell carcinoma. Br. J. Dermatol. 158:527-538.

2. Greenbaum, D., C. Colangelo, K. Williams, and M. Gerstein. 2003. Comparing protein abundance and mRNA expression levels on a genomic scale. Genome Biol. 4:117.

3. Guo, Y., P. Xiao, S. Lei, F. Deng, G.G. Xiao, Y. Liu, X. Chen, L. Li, et al. 2008. How is mRNA expression predictive for protein expression? A correlation study on human circulating monocytes. How Is mRNA Expression Predictive for Protein Expression? a Correlation Study on Human Circulating Monocytes 40:426-436.
4. Wennemers, M., J. Bussink, T. van den Beucken, F.C.G.J. Sweep, and P.N. Span. 2012. Regulation of TRIB3 mRNA and Protein in Breast Cancer. PLoS ONE 7:e49439.

5. Schwanhäusser, B., D. Busse, N. Li, G. Dittmar, J. Schuchhardt, J. Wolf, W. Chen, and M. Selbach. 2011. Global quantification of mammalian gene expression control. Nature 473:337-342.

6. Rotilio, D., A. Della Corte, M. D'Imperio, W. Coletta, S. Marcone, C. Silvestri, L. Giordano, M. Di Michele, and M.B. Donati. 2012. Proteomics: Bases for protein complexity understanding. Thromb. Res. 129:257-262.

7. Sano, T., C.L. Smith, and C.R. Cantor. 1992. Immuno-PCR: very sensitive antigen detection by means of specific antibody-DNA conjugates. Science 258:120-122

8. Sano, T. and C.R. Cantor. 1991. A streptavidinprotein A chimera that allows one-step production of a variety of specific antibody conjugates. Biotechnology (N. Y.) 9:1378-1381.

9. Holland, P.M., R.D. Abramson, R. Watson, and D.H. Gelfand. 1991. Detection of specific polymerase chain reaction product by utilizing the 5"----3" exonuclease activity of Thermus aquaticus DNA polymerase. Proc. Natl. Acad. Sci. USA 88:7276-7280

10. Niemeyer, C.M., M. Adler, and R. Wacker. 2005 Immuno-PCR: high sensitivity detection of proteins by nucleic acid amplification. Trends Biotechnol. 23:208-216.

11. Monjezi, R., S.W. Tan, B.T. Tey, C.C. Sieo, and W.S. Tan. 2012. Detection of hepatitis B virus core antigen by phage display mediated TaqMan real-time immuno-PCR. J. Virol. Methods.

12. Gullberg, M., S.M. Gústafsdóttir, E. Schallmeiner, J. Jarvius, M. Bjarnegård, C. Betsholtz, U. Landegren, and S. Fredriksson. 2004. Cytokine detection by antibody-based proximity ligation. Proc. Natl. Acad. Sci. USA 101:8420-8424.

13. Fredriksson, S., M. Gullberg, J. Jarvius, C. Olsson, K. Pietras, S.M. Gústafsdóttir, A. Östman, and U. Landegren. 2002. Protein detection using proximity-dependent DNA ligation assays. Nat. Biotechnol. 20:473-477.

14. Lundberg, M., S.B. Thorsen, E. Assarsson, A. Villablanca, B. Tran, N. Gee, M. Knowles, B.S. Nielsen, et al. 2011. Multiplexed homogeneous proximity ligation assays for high-throughput protein biomarker research in serological material. Mol Cell Proteomics. 10:M110.004978.

15. Zhu, L., H. Koistinen, P. Wu, A. Närvänen, E. Schallmeiner, S. Fredriksson, U. Landegren, and U.-H. Stenman. 2006. A sensitive proximity ligation assay for active PSA. Biol Chem. 387:769-772.

16. Darmanis, S., R.Y. Nong, M. Hammond, J. Gu, A Alderborn, J. Vanelid, A. Siegbahn, S. Gustafsdottir, et al. 2010. Sensitive Plasma Protein Analysis by Microparticle-based Proximity Ligation Assays. Mol. Cell. Proteomics 9:327-335.

17. Zhang, H.T., J.E. Kacharmina, K. Miyashiro, M.I. Greene, and J. Eberwine. 2001. Protein quantification from complex protein mixtures using a proteomics methodology with single-cell resolution. Proc. Natl. Acad. Sci. USA 98:5497-5502.

18. Freudenberg, J.A., K. Bembas, M.I. Greene, and H. Zhang. 2008. Non-invasive, ultra-sensitive, highthroughput assays to quantify rare biomarkers in the blood. Methods 46:33-38.

19. Chang, B., X. Cheng, S. Yin, T. Pan, H. Zhang, P. Wong, S.-C. Kang, F. Xiao, et al. 2007. Test for detection of disease-associated prion aggregate in the blood of infected but asymptomatic animals. Clin. Vaccine Immunol. 14:36-43.

20. Schweitzer, B., S. Wiltshire, J. Lambert, S. O'Malley, K. Kukanskis, Z. Zhu, S.F. Kingsmore, P.M. Lizardi, and D.C. Ward. 2000. Immunoassays with rolling circle DNA amplification: a versatile platform for ultrasensitive antigen detection. Proc. Natl. Acad. Sci. USA 97:10113-10119.

21. Ding, Y.-Z., Y.-S. Liu, J.-H. Zhou, H.-T. Chen, J. Zhang, L.-N. Ma, and G. Wei. 2011. A highly sensitive detection for foot-and-mouth disease virus by gold nanopariticle improved immuno-PCR. Virol. J. 8:148.

22. Darmanis, S., A. Kähler, L. Spångberg, M Kamali-Moghaddam, U. Landegren, and E. Schallmeiner. 2007. Self-assembly of proximity probes for flexible and modular proximity ligation assays. Biotechniques. 43:443-444.

23. Niemeyer, C.M., M. Adler, B. Pignataro, S. Lenhert, S. Gao, L. Chi, H. Fuchs, and D. Blohm. 1999. Self-assembly of DNA-streptavidin nanostructures and their use as reagents in immuno-PCR. Nucleic Acids Res. 27:4553-4561.

24. Green, N.M. 1965. A spectrophotometric assay for avidin and biotin based on binding of dyes by avidin Biochem. J. 94:23C-24C.

25. Zhou, H., R.J. Fisher, and T.S. Papas. 1993. Universal immuno-PCR for ultra-sensitive target protein detection. Nucleic Acids Res. 21:6038-6039.

26. Chen, L., H. Wei, Y. Guo, Z. Cui, Z. Zhang, and X.-E. Zhang. 2009. Gold nanoparticle enhanced immuno-PCR for ultrasensitive detection of Hantaan virus nucleocapsid protein. J. Immunol. Methods 346:64-70.

27. Fredriksson, S., W. Dixon, H. Ji, A.C. Koong, M. Mindrinos, and R.W. Davis. 2007. Multiplexed protein detection by proximity ligation for cancer biomarker validation. Nat Methods. 4:327-329.

28. Weibrecht, I., K.-J. Leuchowius, C.-M. Clausson, T. Conze, M. Jarvius, W.M. Howell, M. KamaliMoghaddam, and O. Söderberg. 2010. Proximity ligation assays: a recent addition to the proteomics toolbox. Expert Rev. Proteomics 7:401-409.

29. Kim, J., J. Hu, R.S. Sollie, and C.J. Easley. 2010 Improvement of sensitivity and dynamic range in proximity ligation assays by asymmetric connector hybridization. Anal. Chem. 82:6976-6982.

30. Schallmeiner, E., E. Oksanen, O. Ericsson, L. Spångberg, S. Eriksson, U.-H. Stenman, K. Pettersson, and U. Landegren. 2007. Sensitive protein detection via triple-binder proximity ligation assays. Nat. Methods 4:135-137.

31. Niemeyer, C.M.C., B.B. Ceyhan, and D.D. Blohm 1999. Functionalization of covalent DNA-streptavidin conjugates by means of biotinylated modulator components. Bioconjug. Chem. 10:708-719.

32. Kazane, S.A., D. Sok, E.H. Cho, M.L. Uson, P. Kuhn, P.G. Schultz, and V.V. Smider. 2012. Sitespecific DNA-antibody conjugates for specific and sensitive immuno-PCR. Proc. Natl. Acad. Sci. USA 109:3731-3736.

33. Lind, K. and M. Kubista. 2005. Development and evaluation of three real-time immuno-PCR assemblages for quantification of PSA. J. Immunol. Methods 304:107-116

34. Kakizaki, E., T. Yoshida, H. Kawakami, M. Oseto, T. Sakai, and M. Sakai. 1996. Detection of bacterial antigens using immuno-PCR. Lett. Appl. Microbiol. 23:101-103.

35. Hardenbol, P., J. Banér, M. Jain, M. Nilsson, E.A. Namsaraev, G.A. Karlin-Neumann, H. Fakhrai-Rad, M. Ronaghi, et al. 2003. Multi- 
plexed genotyping with sequence-tagged molecular inversion probes. Nat. Biotechnol. 21:673-678.

36. Fredriksson, S., J. Horecka, O. Terje Brustugun, J. Schlingemann, A.C. Koong, R. Tibshirani, and R.W. Davis. 2008. Multiplexed Proximity Ligation Assays to Profile Putative Plasma Biomarkers Relevant to Pancreatic and Ovarian Cancer. Clin. Chem. 54:582-589.

37. Moelans, C.B., R.A. de Weger, M.T. van Blokland, E. van der Wall, and P.J. van Diest. 2010. Simultaneous detection of TOP2A and HER2 gene amplification by multiplex ligation-dependent probe amplification in breast cancer. Mod. Pathol. 23:62-70.

38. Chang, S.T., J.M. Zahn, J. Horecka, P.L. Kunz, J.M. Ford, G.A. Fisher, Q.T. Le, D.T. Chang, et al. 2009. Identification of a biomarker panel using a multiplex proximity ligation assay improves accuracy of pancreatic cancer diagnosis. J. Transl. Med. 7:105.

39. Flanigon, J., M. Kamali-Moghaddam, I. Burbulis, C. Annink, M. Steffen, P. Oeth, R. Brent, D. van den Boom, et al. 2013. Multiplex protein detection with DNA readout via mass spectrometry. $\mathrm{N}$ Biotechnol. 30:153-158.

40. Ueda, H., K. Tsumoto, K. Kubota, E. Suzuki, T. Nagamune, H. Nishimura, P.A. Schueler, G. Winter, et al. 1996. Open sandwich ELISA: a novel immunoassay based on the interchain interaction of antibody variable region. Nat. Biotechnol. 14:17141718

41. Hasan, S., J. Dong, Y. Hara, Y. Morizane, F. Shibasaki, and H. Ueda. 2013. Protein-based Open Sandwich Immuno-PCR for Sensitive Detection of Small Biomarkers. Anal. Sci. 29:871-876.

42. Dong, J., S. Hasan, Y. Fujioka, and H. Ueda. 2012. Detection of small molecule diagnostic markers with phage-based open-sandwich immuno-PCR. J. Immunol. Methods 377:1-7.

43. Ke, R., R.Y. Nong, S. Fredriksson, U. Landegren, and M. Nilsson. 2013. Improving precision of proximity ligation assay by amplified single molecule detection. PLoS ONE 8:e69813.

44. Ellington, A.D. and J.W. Szostak. 1990. In vitro selection of RNA molecules that bind specific ligands. Nature 346:818-822.

45. Tuerk, C. and L. Gold. 1990. Systematic evolution of ligands by exponential enrichment: RNA ligands to bacteriophage T4 DNA polymerase. Science 249:505-510.

46. Baird, G.S. 2010. Where Are All the Aptamers? Am. J. Clin. Pathol. 134:529-531.

47. Dupont, D.M., J.B. Madsen, R.K. Hartmann, B. Tavitian, F. Duconge, J. Kjems, and P.A. Andreasen. 2010. Serum-stable RNA aptamers to urokinase-type plasminogen activator blocking receptor binding. RNA 16:2360-2369.

48. Iliuk, A.B., L. Hu, and W.A. Tao. 2011. Aptamer in Bioanalytical Applications. Anal. Chem. 83:44404452.

49. Ray, P., M.A. Cheek, M.L. Sharaf, N. Li, A.D. Ellington, B.A. Sullenger, B.R. Shaw, and R.R. White. 2012. Aptamer-mediated delivery of chemotherapy to pancreatic cancer cells. Nucleic Acid Ther. 22:295-305

50. Zhang, P., N. Zhao, Z. Zeng, Y. Feng, C.-H. Tung, C.-C. Chang, and Y. Zu. 2009. Using an RNA aptamer probe for flow cytometry detection of CD30-expressing lymphoma cells. Lab Invest. 89:1423-1432.

51. Cerchia, L., C.L. Esposito, S. Camorani, A. Rienzo, L. Stasio, L. Insabato, A. Affuso, and V. de Franciscis. 2012. Targeting Axl with an Highaffinity Inhibitory Aptamer. Mol. Ther. 20:2291-2303.

52. Shi, H., X. He, K. Wang, X. Wu, X. Ye, Q. Guo, W. Tan, Z. Qing, et al. 2011. Activatable aptamer probe for contrast-enhanced in vivo cancer imaging based on cell membrane protein-triggered conformation alteration. Proc. Natl. Acad. Sci. USA 108:3900-3905.

53. Liu, Z., J.-H. Duan, Y.-M. Song, J. Ma, F.-D. Wang, X. Lu, and X.-D. Yang. 2012. Novel HER2 Aptamer Selectively Delivers Cytotoxic Drug to HER2-positive Breast Cancer Cells in Vitro. J. Transl. Med. 10:148.

54. Opazo, F., M. Levy, M. Byrom, C. Schäfer, C. Geisler, T.W. Groemer, A.D. Ellington, and S.O. Rizzoli. 2012. Aptamers as potential tools for superresolution microscopy. Nat Methods. 9:938-939.

55. Djordjevic, M. 2007. SELEX experiments: new prospects, applications and data analysis in inferring regulatory pathways. Biomol. Eng. 24:179-189.

56. Bustin, S.A., V. Benes, J.A. Garson, J. Hellemans, J. Huggett, M. Kubista, R. Mueller, T. Nolan, et al. 2009. The MIQE guidelines: minimum information for publication of quantitative real-time PCR experiments. Clin Chem. 55:611-622.

Received 31 October 2013; accepted 18 February 2014.

Address correspondence to Marcus C Hansen, Aarhus University Hospital, Department of Haematology, Aarhus, Denmark. E-mail: marcus. celik.hansen@ki.au.dk

To purchase reprints of this article, contact: biotechniques@fosterprinting.com

\section{Validate with confidence Optimized for microarray validation}

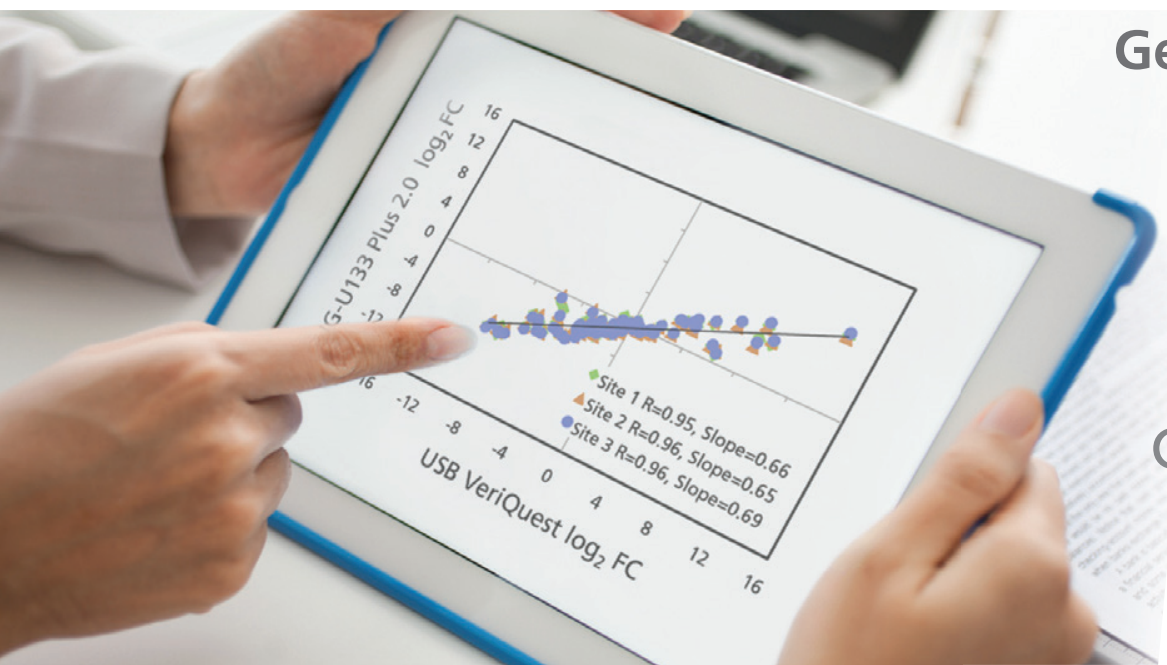

